\title{
THE SZ EFFECT IN THE PLANCK ERA: ASTROPHYSICAL AND COSMOLOGICAL IMPACT
}

\author{
Sergio Colafrancesco ${ }^{a, b, *}$ \\ ${ }^{a}$ University of the Witwatersrand, Johannesburg (South Africa) \\ ${ }^{b}$ INAF-OAR, Monteporzio (Italy) \\ * corresponding author: sergio.colafrancesco@its.ac.za
}

\begin{abstract}
The Sunyaev-Zel'dovich effect (SZE) is a relevant probe for cosmology and particle astrophysics. The Planck Era marks a definite step forward in the use of this probe for astrophysics and cosmology. Astrophysical applications to galaxy clusters, galaxies, radiogalaxies and large-scale structures are discussed. Cosmological relevance for the Dark Energy equation of state, modified Gravity scenarios, Dark Matter search, cosmic magnetism and other cosmological applications is also reviewed. Future directions for the study of the SZE and its polarization are finally outlined.
\end{abstract}

Keywords: cosmology, CMB, dark matter, dark energy, cosmic magnetism, cosmic structures: galaxy clusters, galaxies, radio galaxies.

\section{INTRODUCTION}

Comptonization of the CMB photons by electrons in the plasma confined in the atmospheres of cosmic structures (hereafter referred to as the SZ effect: SZE) is a powerful probe of the energetics, the spectra, and the stratification of their overall electronic distribution because the spectral and spatial characteristics of this process are sensitive to the properties (spectrum, spatial distribution, energy density, pressure) of the electron population. This makes the SZE a powerful astrophysical probe.

Due to its redshift-independent nature, the SZE is also a powerful cosmological probe. To this aim, the SZE in cosmic structures must be determined with very good accuracy in order to derive reliable and unbiased cosmological probes. Therefore, a detailed astrophysical study of the various sources of SZE has to be preliminary fulfilled before their vast cosmological applications are set at work.

\section{The PHYSICS OF THE SZ EFFECT}

The SZE is produced by the inverse Compton scattering (ICS) of CMB photons off the electrons confined in the atmospheres of cosmic structures. Observable quantities of the SZE include:

i) spectral distortions of the $\mathrm{CMB}$ due to upscattering of CMB photons induced by high- $E$ electrons (thermal, non-thermal and relativistic SZE);

ii) spectral distortion of the CMB due to a bulk motion of the electronic plasma w.r.t. the Hubble flow (kinematic SZE);

iii) polarization of the CMB due to dynamical and plasma effects (SZE polarization).

\subsection{THE SZ EFFECT}

The spectral distortion of the CMB spectrum observable in the direction of a galaxy cluster writes [7, 14, 71] as

$$
\Delta I(x)=2 \frac{\left(k T_{\mathrm{CMB}}\right)^{3}}{(h c)^{2}} y g(x),
$$

where $\Delta I(x)=I(x)-I_{0}(x), I(x)$ is the up-scattered CMB spectrum in the direction of the cluster and $I_{0}(x)$ is the unscattered CMB spectrum in the direction of a sky area contiguous to the cluster. Here $x \equiv h \nu / k T_{\mathrm{CMB}}, h$ is the Planck constant, $k$ is the Boltzmann constant, $T_{\mathrm{CMB}}=2.726 \mathrm{~K}$ is the $\mathrm{CMB}$ temperature today and $\nu$ is the observing frequency.

The Comptonization parameter $y$ is

$$
y=\frac{\sigma_{\mathrm{T}}}{m_{\mathrm{e}} c^{2}} \int P_{\mathrm{e}} \mathrm{d} \ell
$$

in terms of the pressure $P_{\mathrm{e}}$ contributed by the electronic population. Here $\sigma_{\mathrm{T}}$ is the Thomson cross section, $m_{\mathrm{e}}$ the electron mass, and $c$ the speed of light.

The spectral function $g(x)$ of the SZE is 14

$$
g(x)=\frac{m_{\mathrm{e}} c^{2}}{\left\langle\varepsilon_{\mathrm{e}}\right\rangle}\left\{\frac{1}{\tau_{\mathrm{e}}}\left[\int_{-\infty}^{+\infty} i_{0}\left(x \mathrm{e}^{-s}\right) P(s) \mathrm{d} s-i_{0}(x)\right]\right\}
$$

in terms of the photon redistribution function $P(s)$ and of

$$
i_{0}(x)=I_{0}(x) /\left[2\left(k T_{\mathrm{CMB}}\right)^{3} /(h c)^{2}\right]=x^{3} /\left(\mathrm{e}^{x}-1\right) .
$$

The quantity

$$
\left\langle\varepsilon_{\mathrm{e}}\right\rangle \equiv \frac{\sigma_{\mathrm{T}}}{\tau_{\mathrm{e}}} \int P_{\mathrm{e}} \mathrm{d} \ell=\int_{0}^{\infty} \mathrm{d} p f_{\mathrm{e}}(p) \frac{1}{3} p v(p) m_{\mathrm{e}} c,
$$




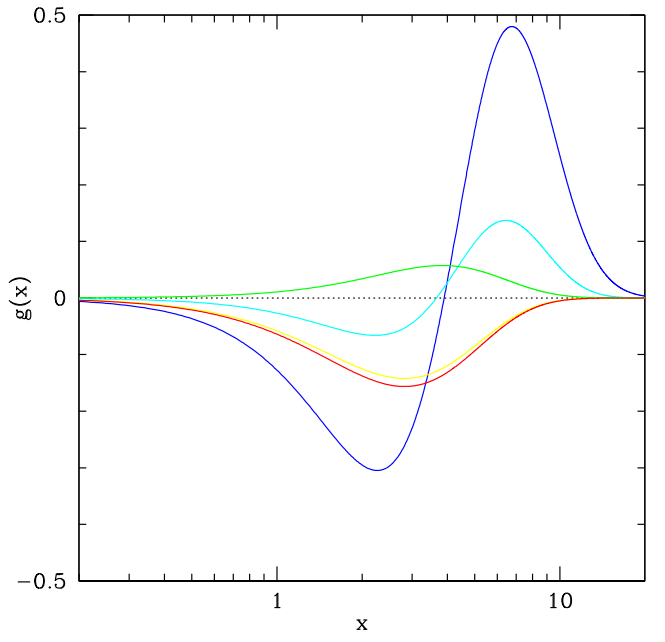

Figure 1. The SZE spectral shape $g(x)$ is shown as a function of the nondimensional frequency $x$ for different electronic populations residing in a cluster: thermal with $k_{\mathrm{B}} T_{\mathrm{e}}=8.2 \mathrm{keV}$ (blue); warm with $k_{\mathrm{B}} T_{\mathrm{e}}=1 \mathrm{keV}$ (cyan); secondary electrons from DM annihilation with $M_{\chi}=20 \mathrm{GeV}$ (red); relativistic electrons which fit the Coma radio halo spectrum (yellow). The kinematic SZE with a negative peculiar velocity (green) is also shown for comparison. The amplitudes of the various curves have been artificially renormalized to highlight their frequency dependence.

where $f_{\mathrm{e}}(p)$ is the normalized electron momentum distribution function, is the average energy of the electron plasma [14.

The optical depth along the line of sight $\ell$ of the electron population with number density $n_{\mathrm{e}}$ is

$$
\tau_{\mathrm{e}}=\sigma_{\mathrm{T}} \int \mathrm{d} \ell n_{\mathrm{e}}
$$

The photon redistribution function $P(s)$, with $s=\ln \left(\nu^{\prime} / \nu\right)$ in terms of the CMB photon frequency increase factor $\nu^{\prime} / \nu$, can be calculated by repeated convolution of the single-scattering redistribution function, $P_{1}(s)=\int \mathrm{d} p f_{\mathrm{e}}(p) P_{\mathrm{s}}(s ; p)$, where $P_{\mathrm{s}}(s ; p)$ depends on the physics of inverse Compton scattering.

The previous description is relativistically covariant and general enough to be applied to both thermal and nonthermal plasma, as well as to a combination of the two (see Fig. 1] and [14, 23, 51, 52] for details).

\subsection{Kinematic SZ EFFeCT}

The velocity (or kinematic) SZE (hereafter kSZE) arises if the plasma causing the thermal, or nonthermal, SZE is moving relative to the Hubble flow. In the reference frame of the scattering particle the CMB radiation appears anisotropic, and the effect of the ICS is to re-isotropize the radiation slightly. Back in the rest frame of the observer the radiation field is no longer isotropic, but shows a structure towards the scattering atmosphere with amplitude $\propto \tau_{\mathrm{e}} V_{\mathrm{t}} / c$, where $V_{\mathrm{t}}$ is the component of the peculiar velocity of the scattering atmosphere along the line of sight [66, 70].
The brightness change of the CMB due to the kSZE is given by

$$
\frac{\Delta I}{I}=-\tau_{\mathrm{e}} \beta_{\mathrm{t}} \frac{x \mathrm{e}^{x}}{\mathrm{e}^{x}-1}
$$

with $\beta_{\mathrm{t}} \equiv \frac{V_{\mathrm{t}}}{c}[54,70]$. A general relativistic description of the $\mathrm{kSZE}$ has been given in the framework of the general Boltzmann equation [40] and in the relativistic covariant formalism [51, 52.

\subsection{SZE POLARIZATION}

The ICS process yields naturally a polarized upscattered radiation field (see, e.g., [10] and references therein). The polarization $\Pi$ of the SZE arises from various dynamical and plasma effects [5, 27, 44, 68]: galaxy clusters transverse motion $\left(\Pi_{\mathrm{k}} \propto \beta_{\mathrm{t}}^{2} \tau\right.$ in the Rayleigh-Jeans, RJ, regime), transverse motions of plasma within the cluster $\left(\Pi_{\mathrm{v}} \propto \beta_{\mathrm{t}} \tau^{2}\right.$ in the RJ regime) and multiple scattering between electrons and CMB photons within the cluster $\left(\Pi_{\text {th }} \propto \Theta \tau^{2}\right.$ in the RJ regime for the thermal SZE with $\left.\Theta=k T_{\mathrm{e}} / m_{\mathrm{e}} c^{2}\right)$. A general, covariant, relativistic derivation of the SZE polarization for thermal, non-thermal and relativistic plasma can be derived [27] and generalizes the non-relativistic derivation 68 in a way similar to the general derivation of the SZE [14] previously discussed.

\section{AstrophysicAl AND \\ COSMOLOGICAL IMPACT}

Studying the SZE in various cosmic atmospheres provides many insights on their energetics, pressure and dynamical structure. The combination of SZE with other emission mechanisms related to the same particle distribution (i.e., synchrotron, high- $E$ ICS emission, bremsstrahlung emission) provides further information on the radiation, matter and magnetic fields that are co-spatial with the electrons producing the SZE. These properties of the SZE concern various cosmic structures, from galaxy clusters to radiogalaxy lobes, from galaxy halos to supercluster and the WHIM (see Sect. 6 below).

The redshift-independent nature of the SZE allow to use this effect as a powerful cosmological probe by using both the redshift evolution of cluster abundance and direct probes of cosmological parameters. The SZE has a wide range of cosmological applications: it can be used to determine the main cosmological parameters and the Dark Energy (DE) equation of state, and also set constraints to modified Gravity scenarios and to the properties of primordial magnetic fields (see Sect. 7 below).

Observations of the SZE in cosmic structures have been performed in the last two decades with increasing sensitivity and spatial resolution but in the limited frequency bands accessible from the ground, and the availability of the Planck surveyor is now opening its study over a wider frequency range. We discuss in the following the main achievements on the SZE physics in the pre-Planck era and in the Planck era, 
and we also outline some of the future possibilities of exploiting the large amount of astrophysical and cosmological information contained in the SZE.

\section{The PAST: PRE-PlanCK ERA}

The SZE has been searched in galaxy clusters since it was originally proposed [70, 71] using various techniques. Three distinct techniques for the measurement of the thermal SZE in clusters of galaxies yielded reliable results: single-dish radiometric observations, bolometric observations, and interferometric observations (we refer to [7] for a discussion of the weaknesses and strengths of each technique and the types of systematic error from which they suffer). No concerted results for measuring the polarization SZE have yet been obtained.

The relevant milestones of the most relevant SZE observations before the Planck Era are listed below:

- 1983. The Owens Valley Radio Observatory (OVRO) first detect the SZE at $30 \mathrm{GHz}$ from clusters of galaxies.

- 1993. The Ryle Telescope is the first telescope to image a cluster of galaxies in the SZE.

- 1998. The first sub-mm observation of the SZE effect was obtained by the PRONAOS $2 \mathrm{~m}$ stratospheric telescope towards Abell 2163 [43].

- 2001. The first four band spectrum of the SZE was obtained for the Coma cluster with the MITO $3.5 \mathrm{~m}$ telescope [32.

- 2003. The WMAP spacecraft maps the CMB over the whole sky with some (limited) all-sky sensitivity to the SZE.

- 2005. The Atacama Pathfinder Experiment (APEX) SZE camera saw first light and shortly after began pointed observations of galaxy clusters.

- 2005. The Arcminute Microkelvin Imager (AMI) and the SZ Array (SZA) each begin surveys for very high redshift clusters of galaxies using the SZE.

- 2007. The South Pole Telescope (SPT) saw first light on February 16, 2007, and began science observations in March of that same year.

- 2007. The Atacama Cosmology Telescope (ACT) saw first light on June 8, 2007, and began an SZE survey of galaxy clusters.

- 2008. The SPT discover for the first time galaxy clusters in blind-survey mode via the SZE.

- 2012. The ACT find statistical evidence for the kSZE [38.

The pioneering era of the SZE study has been dotted with numerous technological and scientific successes.

Ground-based SZE experiments (e.g., SPT, APEX, ACT, AMI, GBT, among others) provided excellent results in terms of imaging and blind search surveys with their low frequency, multiple-band observations,

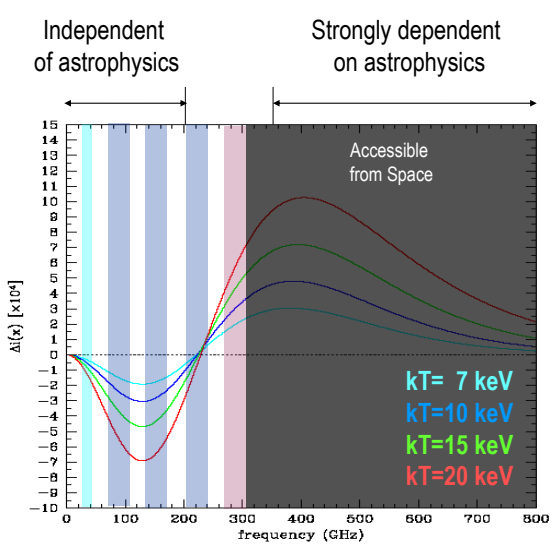

FIgURE 2. The thermal SZE spectrum of typical galaxy clusters for different plasma temperatures (as indicated) and the same value of the optical depth. The low- $\nu$ part $(\nu \lesssim 220 \mathrm{GHz})$ of the spectrum depends mostly on the total Compton parameter $y \propto \int \mathrm{d} \ell P_{\text {tot }}$ and there is no strong spectral dependence on the temperature. The high- $\nu$ part of the spectrum (at $\gtrsim 300 \mathrm{GHz}$ ) shows a strong spectral dependence from the plasma temperature 23. The usual frequency bands where the SZE is observed from the ground are shown as blue-cyan bands, while the region accessible only from space observations is shown by the gray shaded area.

but they do not have neither true spectroscopic capabilities nor a wide spectroscopic frequency band, and they are not sensitive to the high- $\nu$ range ( $\lesssim 400 \mathrm{GHz}$ ) of the SZE signals, which is crucial to exploit the astrophysical information contained in the SZE (see Figs. 1 and 2). The region around the null of the thermal SZE, i.e. at $\sim 220 \mathrm{GHz}$ is rich in astrophysical information because the frequency location of the null of the SZE is directly dependent on the total pressure of the plasma [14] but this frequency diplacement is rather difficult to be measured due to the low amplitude of the SZE signal at these frequencies and to the heavy biases and uncertainties (due to the level of the CMB subtraction, the kinematic SZE, and possible sources of non-thermal SZE) in the measurement (see [23, 31]).

In fact, ground-based instruments widely improved the source statistics (crucial to obtain cosmological information using the SZE) and the angular resolution of SZE images (crucial to disentangle the extended SZE signal from point-source contamination), but add little to the physical specification of the detected SZE sources, and therefore they need X-ray and optical follow-up to determine the characteristics of the physical parameters extracted from SZE observations.

\section{The PResent: Planck erA}

The Planck surveyor satellite was launched in May 2009, together with the Herschel satellite, and set in an L2 orbit. Planck has a $1.5 \mathrm{~m}$ gregorian telescope 
and receivers covering 9 frequency bands, from 30 to $857 \mathrm{GHz}$. The angular resolution of the experiment goes from $\sim 5$ (at high- $\nu$ ) to 30 (at low- $\nu$ ) arcmin. The two main experiments on board Planck are the LFI with 22 radiometers, covering the 3 low- $\nu$ frequency bands, and the HFI with 72 bolometers plus thermometers cooled down to $0.1 \mathrm{~K}$, covering the higher 6 frequency bands. The nominal mission consists of 2 full sky surveys and the extended mission of 4 surveys at least.

The Planck early SZE science observations yielded 189 SZE sources with $\mathrm{S} / \mathrm{N}>6$ which provides the first SZE measure for $\sim 80 \%$ of the known galaxy clusters, and 20 additional new clusters discovered by Planck via the SZE (see 61 on behalf of the Planck Collaboration for a recent discussion on the detection, follow-up and validation procedures). This is the largest sample so far of SZE detected clusters. Planck detected SZE clusters are followed-up with a multi-frequency observation program in the X-rays (XMM-Newton), SZE (AMI), optical (ENO, ESO, RTT, NOT, NOAO, among others) to obtain confirmation, redshift estimation and estimates of the global physical parameters. Planck results show that the SZE selection is a very powerful method for the detection of new distant and very massive clusters. Planck is also unveiling a population of dynamically perturbed clusters at $z \gtrsim 0.3$, possibly underrepresented in X-ray surveys. The information collected so far strengthen our overall view of the ICM properties and mass content in galaxy clusters and is getting to close the long standing issue of the "missing hot baryons" from the excellent agreement between observed the SZE Compton parameter $Y_{\text {SZE }}$ and X-ray-based predictions.

Most of these results are discussed in the early and intermediate papers [55 60] and more detailed analyses are still coming.

The Herschel satellite (co-eval with Planck) has been able to observe the SZE in a few pointed clusters with the SPIRE instrument equipped with an FTS spectrometer working in the frequency range $\sim 600 \div 1200 \mathrm{GHz}$. The possibility to have sensitive spectroscopic measurements in these high-frequency bands opens the way to the deep astrophysical exploitation of the SZE. As an example, two additional data points on the SZE spectrum of the Bullet cluster observed with Herschel-SPIRE [81] allowed immediately to establish a number of properties for the thermal and non-thermal plasma superposition in the atmosphere of this strong merging cluster (see, e.g. [24, 63, 64]. This has been possible because the access to the very high- $\nu$ part of the SZE spectrum contains detailed information on the relativistic effect on the single thermal plasma and on the presence of additional plasmas of either thermal or non-thermal nature (see discussion in [24, 26]).

Planck and Herschel observations of the SZE are in fact opening a rich field of investigation that will fully blossom in the next years with the full exploitation of spatially-resolved spectroscopic SZE observations. In the following Sect. 6 we discuss some of the astrophysical studies possible with the SZE and in Sect.7 we will address their cosmological relevance.

\section{AstrophysicAl IMPACT}

Studying the SZE in various cosmic atmospheres provides many insights on their energetics, pressure and dynamical structure. The combination of SZE with other emission mechanisms related to the same particle distribution (i.e., synchrotron, high- $E$ ICS emission, bremsstrahlung emission) provides further information on the radiation, matter and magnetic fields that are co-spatial with the electrons producing the SZE. These properties of the SZE concern various cosmic structures.

\subsection{Galaxy Clusters}

Galaxy clusters are the largest containers of Dark Matter (DM), diffuse hot (and probably also warm) thermal plasma as probed by X-ray emission from the ICM, cosmic rays and diffuse non-thermal plasma as indicated by diffuse radio emission, $\sim \mu \mathrm{G}$ amplitude magnetic fields as indicated by diffuse radio emission and Faraday rotation measures, they have cool cores likely heated by cosmic rays ejected and/or accelerated in the radiogalaxy $(\mathrm{RG})$ jet/lobes, whose late evolution might produce large ICM cavities filled with relativistic or non-thermal plasma 21.

The SZE spectra of the various electron populations (see Fig. 1) show quite different shapes that reflect the different electron spectra and pressure (energy density), and their analysis can be used to disentangle the plasma stratification of the cluster atmosphere.

Precise observations of the SZE at microwave and mm wavelengths are crucial for unveiling the detailed structure of cluster atmospheres, their temperature distribution, and the possible presence of suprathermal and/or nonthermal plasma because the high-frequency part (i.e. at $\nu \gtrsim 350 \mathrm{GHZ}$ or $x \gtrsim 6$ ) of the SZE spectrum is more sensitive to the relativistic effects of the electron momentum distribution [14, 19, 23. This is even more so for galaxy clusters with a complex plasma distribution as found for powerful merging clusters, like the exemplary case offered by the Bullet cluster (1ES0657-56) [24].

Powerful merging events in galaxy clusters can, in fact, produce an additional high- $T$ plasma distribution (if the electron acceleration time scale at the merging shocks is longer than their equilibration time scale 78 ), or an additional nonthermal population (produced either in a merging process with a very short acceleration time scale or by secondary electrons produced by $\mathrm{p}-\mathrm{p}$ collisions, after the high- $E$ protons have been accelerated by the merging and accumulate in the cluster region on long time scales [79]).

The quasi-stationary case provided by the competition between particle thermalization and stochastic acceleration and momentum diffusion 35] can develop 


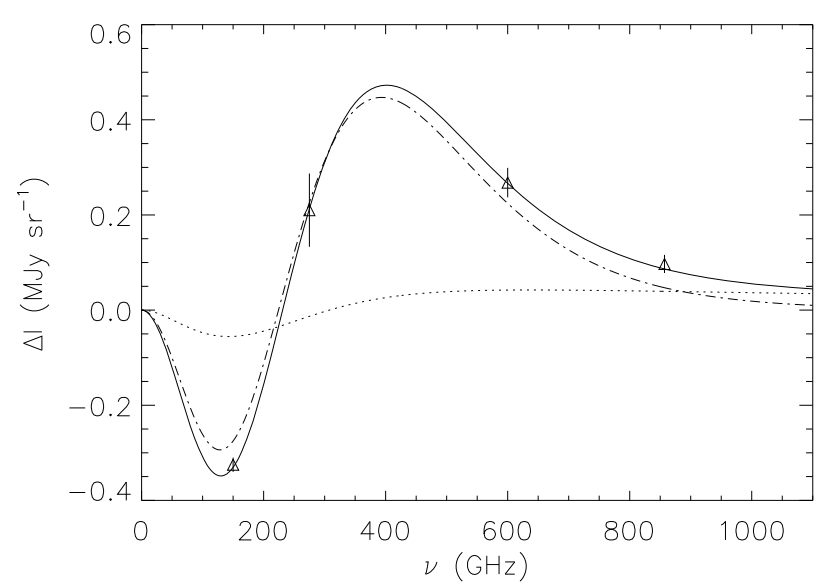

Figure 3. The SZE spectrum at the Bullet cluster center modeled with a thermal plus nonthermal plasma: thermal plasma with $k T=13.9 \mathrm{keV}$ and $\tau=1.1 \times 10^{-2}$ (dot-dashed); nonthermal plasma with $p_{1}=1, s=2.7$ and $\tau=2.3 \times 10^{-4}$ (dotted); total SZE produced by the sum of the two plasmas (solid).

a subrelativistic electron distribution tail and can produce suprathermal (or nearly nonthermal) regions in the cluster atmosphere.

A quantitative estimate of the temperature inhomogeneity (stratification) along the line of sight is possible using SZE data only providing a measure the temperature standard deviation of the cluster plasma along the line of sight. We found that the Bullet cluster has a temperature standard deviation of $10.6 \pm 3.8 \mathrm{keV}$ 65]. This result (obtained for the first time with SZE measurements) shows that the temperature distribution in the Bullet cluster is strongly inhomogeneous and provides a new method for studying galaxy clusters in depth. Study of the multifrequency (from $\sim 30$ to $\sim 850 \mathrm{GHz}$ ) SZE signal observed in the Bullet cluster shows, in fact, the presence of a thermal plasma at $\sim 13.9 \mathrm{keV}$ coexisting with a second plasma component, either at higher temperature $(\approx 25 \mathrm{keV})$ or, more preferably, of a nonthermal origin [24] (see Fig 3). Additional observations of the Bullet cluster at $\nu \sim 400 \mathrm{GHz}$ with a precision $\lesssim 1 \%$ of the expected signal will be able to further distinguish between the two cases of non-thermal power-law or suprathermal tail [24].

SZE observations over a wide frequency range, and especially with high sensitivity in the high- $\nu$ range, can also add relevant information on the electron distribution function (DF) in the ICM, a subject that even though relevant for a proper analysis of the SZE - has not been addressed in details so far. The relativistic kinetic theory, on which the DF derivation is based, is still a subject of numerous debates (see discussion in [63]).

SZE observations can separate the SZE spectrum caused by a departure from the diffusive approximation based on the Kompaneets approach [42 from those which are due to using a relativistic correct DF instead of a Maxwell-Boltzman DF (see Fig. 4)

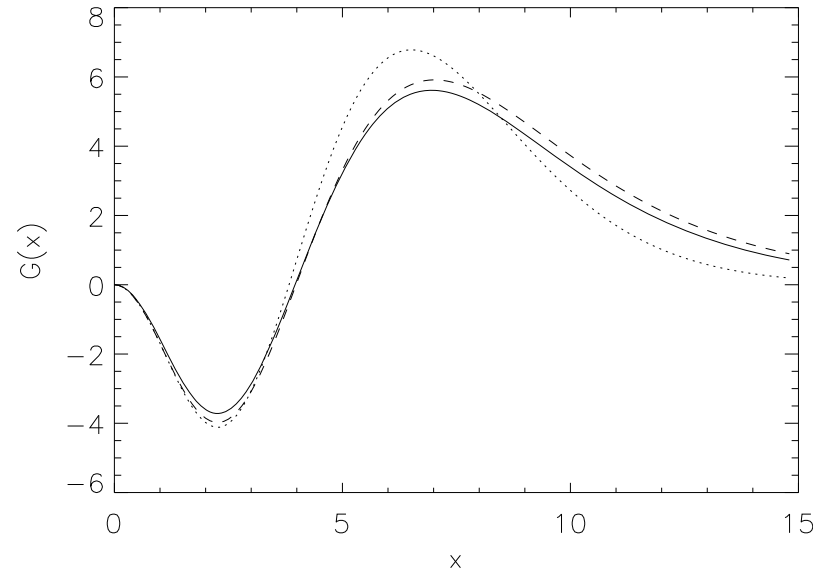

Figure 4 . The SZE intensity spectra for a massive cluster with a temperature of $k T_{\mathrm{e}}=15.3 \mathrm{keV}$ for Juttner (solid) and Maxwell-Boltzman (dashed) DFs. The non-relativistic SZE spectrum (solid) is also shown for comparison. Figure from 63 .

and therefore set constraints to the actual electron DF 63 .

This analysis is best fulfilled in hot massive clusters because the SZE intensity change due to using a relativistic correct DF instead of a Maxwell-Boltzman DF are much larger in hot clusters due to the fact that relativistic SZE corrections scale as $\propto T^{5 / 2}$. A method used to derive the DF of electrons using SZE multifrequency observations of massive hot clusters [63] makes use of Fourier analysis representation of the approximate electron DF whose parameters are best fitted using observations in the (optimal) frequency channels at $375,600,700,857 \mathrm{GHz}$.

A morphological analysis of the SZE observed at various frequencies adds relevant information to assess the pressure and energy density structure of cluster atmospheres. Morphological SZE differences are particularly evident for clusters undergoing violent mergers that create large inhomogeneities of the electron DF.

SZE intensity maps of merging clusters obtained from hydrodynamical simulations show that the morphology of the SZE intensity maps observable with LABOCA (at $345 \mathrm{GHz}$ ) and Herschel-SPIRE (at $857 \mathrm{GHz}$ ) are rather different 64 (see Fig. 5). For a Bullet-like cluster, the SZE intensity map at $857 \mathrm{GHz}$ has a spatial feature caused by the presence of the cold Bullet-like substructure seen also in the X-ray surface brightness map. However, this cold substructure is not present in the SZE intensity map at $345 \mathrm{GHz}$. This is a consequence of the relativistic effects of the SZE and shows that observations of the SZE intensity maps at very high frequencies can reveal complex pressure substructures within the atmospheres of massive galaxy clusters.

For the cluster A2219, the SZE intensity map at $857 \mathrm{GHz}$ (obtained by using the Chandra density and temperature maps [49]) shows evidence for a largescale shock heated region and a very hot region coinci- 


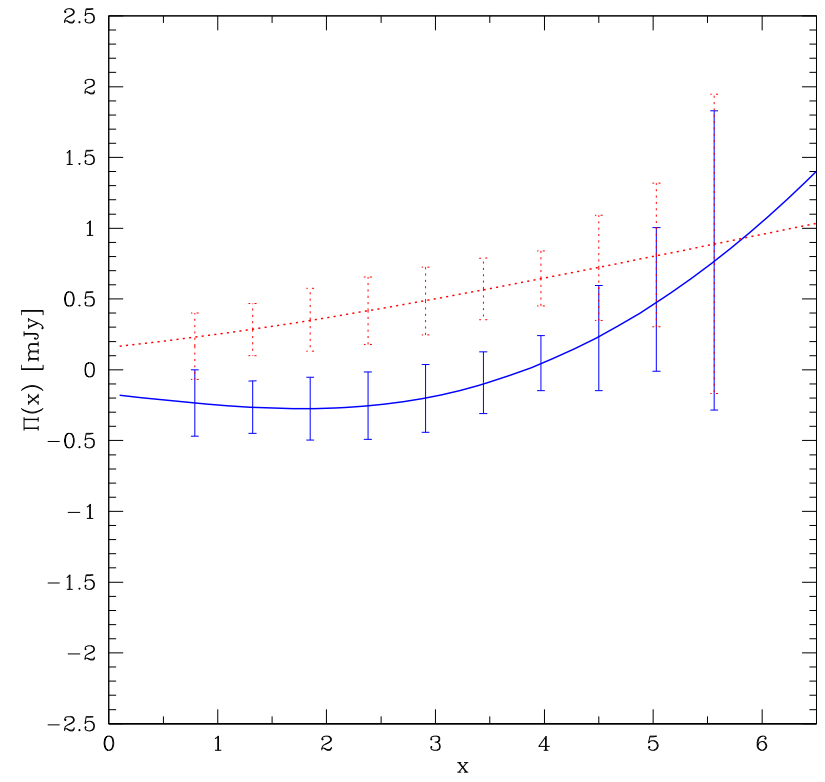

FIgURE 6. The thermal SZE polarization spectrum $\Pi_{\mathrm{th}}$ (blue solid) from a stacking analysis of 20 clusters with $k T>10 \mathrm{keV}$ and $\tau>0.03$ is compared with the same analysis for the kSZE polarization spectrum $\Pi_{\mathrm{k}}$ (red dotted). The statistical uncertainties refer to a stacking analysis produced with COrE [29].

dent with the peak of the X-ray surface brightness (see Fig. 5). This result shows that the analysis of the SZE signal at $857 \mathrm{GHz}$, correlated with lower- $\nu$ observations offers a promising method for unveiling high- $T$ regions in massive merging clusters using available experiments like, e.g LABOCA and Herschel-SPIRE.

In more relaxed clusters spectroscopic measurements of the SZE over a wide frequency band allow to derive precise information on the temperature distribution and on the cool-core nature independently of X-ray priors 23 ] and hence reconstruct the full set of cluster physical parameters 31].

Polarization measurement of the (thermal and nonthermal) SZE are able to add further information on the transverse plasma motions within the cluster and on the pressure substructure of the plasma.

SZE polarization signals in galaxy clusters are quite low and typically below mJy (or $\mu \mathrm{K}$ ) level even for high- $T$ clusters (see Fig. 6).

It is interesting, however, to note that the SZE polarization in cluster has quite different spectra w.r.t. the intensity SZE spectrum.

Combining intensity and polarization observations of the SZE can uncover unique details of the $3 \mathrm{~d}$ (projected and along the line of sight) velocity structure of the ICM, of its $3 \mathrm{~d}$ pressure structure and of the influence of a structured magnetic field in the stratification of the ICM, and therefore provides a full tomography of cluster atmospheres. Analogously, the combination of the intensity and polarization observations of the kinematic SZE (and its frequency dependence) can yield crucial information on the $3 \mathrm{~d}$ distribution of the cosmological velocity field traced by galaxy clusters.
Specifically, the ratio $\Delta I_{\mathrm{th}} / \Pi_{\mathrm{th}}$ yields direct information on the plasma optical depth $\tau$, and the ratio $\Delta I_{\mathrm{th}} / \Pi_{\mathrm{v}}$ on the combination $\tau \beta_{\mathrm{t}}$, thus allowing to use intensity and polarization SZE measurements to fully disentangle the pressure and velocity structure of the cluster atmospheres.

SZE polarization measurements are quite difficult to obtain with present-day experiments and they are also at the limit of next generation experiments. The required sensitivity to disentangle these signals at a high $(\sim 3 \sigma)$ confidence level should be of order of order $\sim 10 \mu \mathrm{Jy}$. However, stacking analysis of even small samples (order of $\sim 20$ ) of hot and dense galaxy clusters observed at multifrequency would allow to determine statistically the polarization signals of the thermal SZE for clusters with $k T>10 \mathrm{keV}$ and $\tau>0.03$ (see Fig. 6) in the optimal frequency range $\approx 90 \div 250 \mathrm{GHz}$.

\subsection{Cluster Cavities}

The atmospheres of galaxy clusters often show the presence of bubbles filled with high- $E$ particles and magnetic field that are sites of bright radio emission and produce cavities in the cluster X-ray emission.

Cavities with diameters ranging from a few to a few hundreds of kpc have been observed by Chandra in the X-ray emission maps of several galaxy clusters and groups [8, 47]. While the properties of these cavities and of the relativistic plasma they contain is usually studied by combining X-ray and radio observations, an alternative and efficient strategy is to study the consequences of the SZE produced by the high-energy electrons filling the cavities [16, 53] whose amplitude, spectral and spatial features depend on the overall pressure and energetics of the relativistic plasma in the cavities.

As an example, the overall SZE observable along the line of sight (LOS) through a cluster containing cavities (see Fig. 7 for the case of the cluster MS0735.6+7421) is the combination of the nonthermal SZE produced by the cavity and of the thermal SZE produced by the surrounding ICM.

Due to the different $\nu$-dependence of the thermal and non-thermal SZE, the non-thermal SZE from a cluster cavity shows up uncontaminated at frequencies $\nu \approx 220 \mathrm{GHz}$ : at this frequency, in fact, the overall SZE from the cluster reveals only the ICS of the electrons residing in the cavities without the presence of the intense thermal SZE dominating at lower and higher frequencies. The cavity's SZE becomes dominant again at very high- $\nu(x \gtrsim 14$ or $\nu \gtrsim 800 \mathrm{GHz})$ where the nonthermal electrons dominate the overall ICS emission (see Fig. 7).

The cavity's SZE is more spatially concentrated than the overall cluster SZE because it is only emerging from the cavity regions: this fact allows to study the overall energetics and pressure structure of the cavity's high- $E$ particle population and the $B$-field 

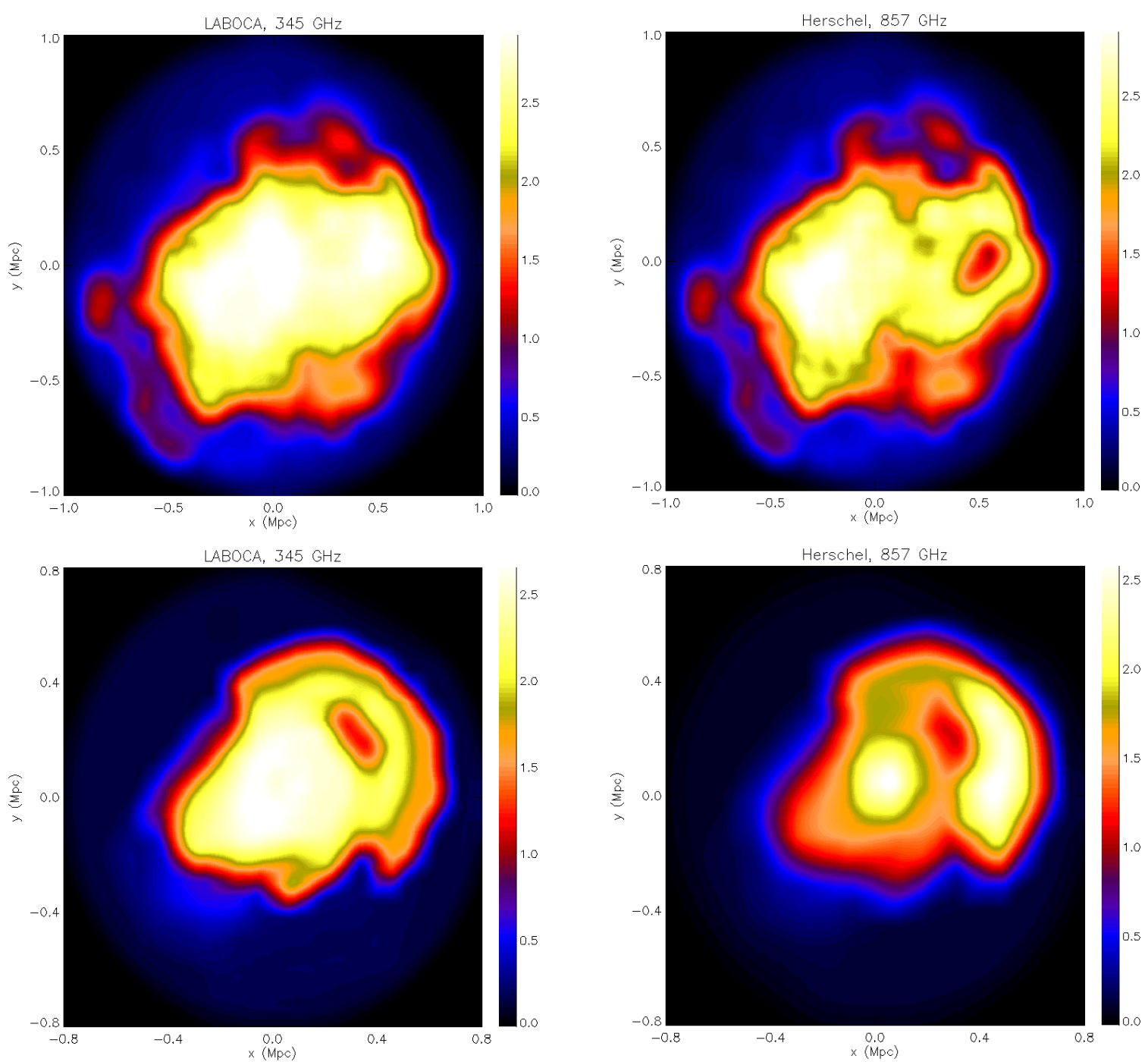

Figure 5. From top to bottom. The SZE signal to noise ratio map for the cluster 1E0657-558 at $345 \mathrm{GHz}$ smoothed to the resolution of LABOCA and at $857 \mathrm{GHz}$ smoothed to the resolution of Herschel-SPIRE. Analogous maps for the cluster A2219. Figures from 64].

structure in combination with $\mathrm{X}$-rays and radio images.

The observation of the crossover of the non-thermal SZE from the cavities (which depends on the value of $E_{\min }\left(p_{1}\right)$ or, equivalently, on the value $\left.P_{\text {cavity }}\right)$ provides a way to determine the total pressure and hence the nature of the electron population within the cavity [16], an evidence which adds crucial, complementary information to the $\mathrm{X}$-ray and radio analysis.

Alternative studies have been performed by assuming that cluster cavities contain a high- $T$ plasma $\left(\sim 10^{9} \div 10^{10} \mathrm{~K}\right) 62$. In this case the SZE flux from cocoons in the central part of a distant elliptical and a nearby galaxy cluster are of the same order. For a high$T$ plasma, the cocoon's SZE spectrum is rather flat at high- $\nu$ resembling the shape of the non-thermal SZE from cavities. In this high- $T$ plasma model, however, no strong radio emission at $\nu \gtrsim 1 \mathrm{GHz}$ (as instead observed) is expected from the cocoon, unless the cocoon's $B$-field is very high $B \gtrsim 10^{3} \mu \mathrm{G}$.

\subsection{RADIOGALAXY LOBES}

Studies of (giant) radio-galaxy (RG) lobes (see, e.g., [9, 30, 39, 41, and references therein) have shown that these extended structures contain relativistic electrons that are currently available to produce both low- $\nu$ synchrotron radio emission and ICS of the CMB (as well as other radiation background) photons. As a consequence, an SZE from the lobes of RGs is inevitably expected [20]. Such non-thermal, relativistic SZE has a specific spectral shape that depends on the shape and energy extent of the spectrum of the electrons residing in $\mathrm{RG}$ lobes.

The SZE emission from RG lobes is expected to be co-spatial with the relative ICS X-ray emission [20] and its spectral properties are related to those of the relative ICS X-ray emission. In fact, the spectral slope of the ICS X-ray emission $\alpha_{\mathrm{X}}=(\alpha-1) / 2$ (where $F_{\mathrm{ICS}} \sim E^{-\alpha \mathrm{x}}$ ) can be used to set the electron energy spectral slope $\alpha$ (where $N_{\mathrm{e}} \sim E^{-\alpha}$ ) necessary to compute the SZE spectrum, and to check its 

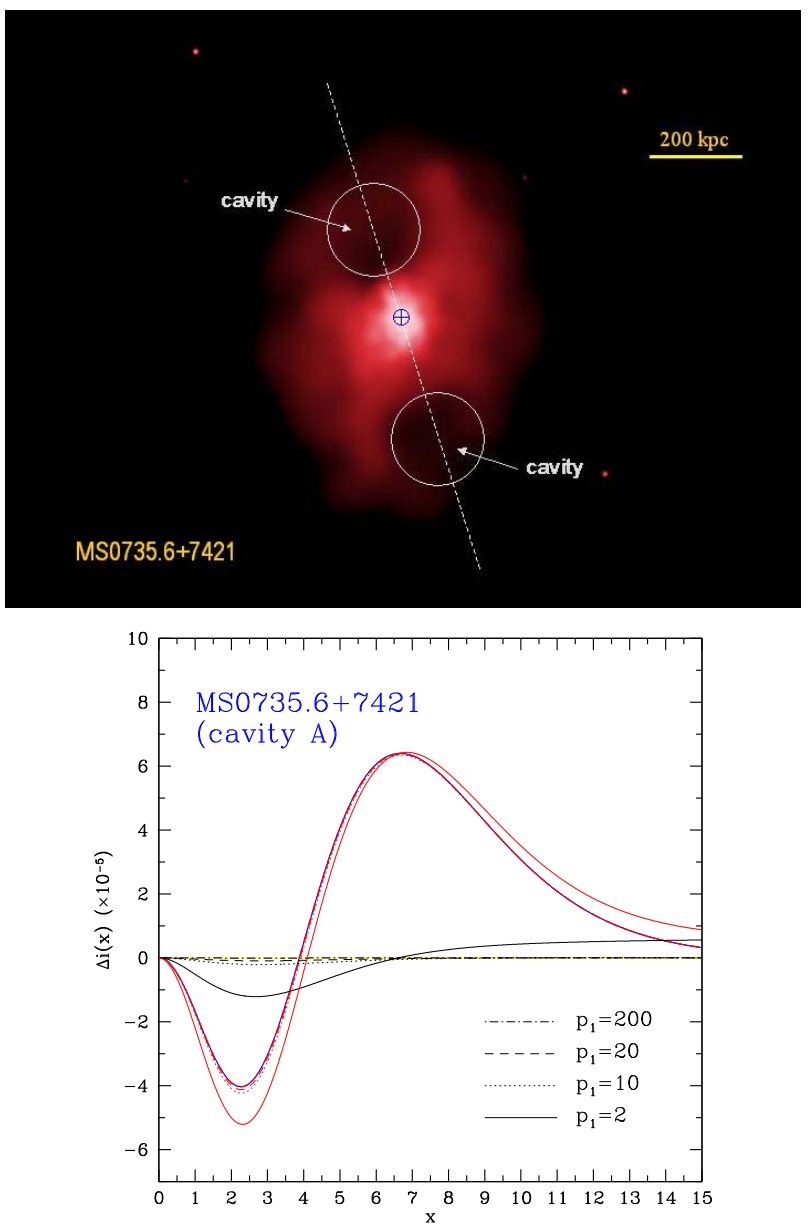

Figure 7. Top. The geometry of the cavities in the cluster MS0735.6+7421. Bottom. The SZE spectrum has been computed at a projected radius of $\approx 125 \mathrm{kpc}$ from the cluster center where the LOS passes through the center of northern cavity. The thermal SZE (blue), the non-thermal SZE from the cavity (black) and the total SZE (red) are shown. The non-thermal SZE is normalized to the cavity pressure $P=6 \times 10^{-11} \mathrm{erg} \mathrm{cm}^{-3}$, and is shown for various values of $p_{1}$. Figure from [16].

consistency with the synchrotron radio spectral in$\operatorname{dex} \alpha_{\mathrm{r}}=(\alpha-1) / 2$ (where $\left.F_{\text {synch }} \sim E^{-\alpha_{\mathrm{r}}}\right)$, that is expected to have the same value [20, 25].

The SZE in RG lobes has not been detected yet: only loose upper limits have been so far derived on the SZE from these sources (see [7] for a review; see also a recent attempt [80] to detect this effect at radio wavelengths in the giant radio galaxy B1358+305).

A detection of the SZE from RG lobes can provide a determination of the total energy density and pressure of the electron population in the lobes [20] allowing to determine the value of $E_{\min }$ once the slope of the electron spectrum is determined from radio and/or X-ray observations. SZE measurements provide a much more accurate estimates of the electron pressure/energy density than with other technique like ICS X-ray emission or synchrotron radio emission, since the former can only provide an estimate of the electron energetics in the high-energy part of the electron spectrum, and the latter is sensitive to the degenerate combination of the electron spectrum and of the magnetic field in the radio lobes.

The combination of SZE observations (that depend on the electron distribution and on the known CMB radiation field) and the radio observations (which depend on the combination of the electron distribution and of the magnetic field distribution) provides an unbiased estimate of the overall $B$-field in the lobe by using the ratio $F_{\text {radio }} / F_{\mathrm{SZE}} \approx \mathcal{E}_{B} / \mathcal{E}_{\mathrm{CMB}}$, that is more reliable than that obtained from the combination of ICS X-ray (or gamma-ray) and radio emission [25].

The spatially resolved study of the SZE and synchrotron emission in RG lobes also provide indication on the radial behaviour of both the leptonic pressure and of the magnetic field from the inner parts to the boundaries of the lobes.

The study of the pressure evolution in RG lobes can provide crucial indications on the transition from radio lobe environments to the atmospheres of giant cavities observed in galaxy cluster atmospheres (see, e.g., [48] for a review), which seem naturally related to the penetration of RG jets/lobes into the ICM (see Fig. 77.

A substantial SZE polarization is also expected in RG lobes due to both coherent transverse motions of the plasma along the jet/lobe direction and to the electron pressure substructures induced by e.g. plasma inhomogeneities and magnetic field turbulence. The transverse velocity-induced polarization is $\Pi_{\mathrm{v}} \propto \tau_{\text {rel }}\left(\beta_{\mathrm{t}} \tau_{\text {rel }}\right)$, and the multiple scattering induced polarization is $\Pi_{\tau} \propto \tau_{\text {rel }} P_{\text {rel }}$ where $P_{\text {rel }}$ is the pressure of the relativistic electron distribution. Observations of the SZE and its polarization in RG lobes can yield, therefore, direct information on electrons $\tau_{\text {rel }}$ and $\beta_{\mathrm{t}}$ in the RG lobe.

\subsection{Galaxies}

Hot gas trapped in a DM halo can produce a SZE. A typical galaxy halo might hence show an integrated thermal SZE at the level of $\lesssim 0.5 \mathrm{mJy} \operatorname{arcmin}^{-2}$ from a plasma with $T \sim 10^{6} \mathrm{~K}$ and density $n_{\mathrm{e}} \sim 10^{-3} \mathrm{~cm}^{-3}$ extended for $\sim 50 \mathrm{kpc}$ in the inter-stellar medium (ISM). Due to this fact, it has been claimed that the halo of M31, may be one of the brightest integrated SZE sources in the sky [73: for various realistic gas distributions consistent with current X-ray limits, the integrated SZE decrement from M31 could be comparable to decrements already detected in more distant sources, provided that its halo contains an appreciable quantity of hot gas. A measurement of galaxy halo SZE would provide direct information on the mass, spatial distribution and thermodynamic state of the plasma in a low-mass galactic halo, and could place important constraints on current models of galaxy formation. Detecting such an extended, low-amplitude signal will be challenging, but possible with all-sky SZE maps from Planck. 
An SZE is also expected from galaxy outflows swept by galaxy (hyper)winds. A thermal SZE is expected to arise from the shocked bubble plasma in a strong galaxy wind described by a simple, spherical blast wave model 67.

However, such simple recipe for the SZE from galaxy winds is to be modified by the presence of cosmic rays and magnetic field in the expanding wind 28] leading on one side to a more complex SZE spectrum, and on the other side to an amplification of the overall SZE at high frequencies, thus increasing the detection probability. SZE observations from galaxy winds will be possible with high-sensitivity and high-resolution telescopes like ALMA and SKA due to its low amplitude and spatial extension.

\subsection{Superclusters AND the WHIM}

On the very large scales of the universe the SZE can be used to trace the distribution of baryons in the supercluster environment and in the Warm Hot Intergalactic Medium (WHIM).

A detection at $33 \mathrm{GHz}$ of a strong temperature decrement in the CMB towards the core of the Cornoa Borealis supercluster has been found [37. Multifrequency observations with VSA and MITO suggest the existence of a thermal SZE component in the spectrum of this cold spot with $y=7.8_{-5.3}^{+4.4} \times 10^{-6}$ [4], which would account for roughly $25 \%$ of the total observed temperature decrement towards this supercluster.

N-body MareNostrum Universe SPH simulations have been further used to study the thermal and kSZE associated with superclusters of galaxies, and in particular, superclusters with characteristics (i.e., total mass, overdensity and number density of cluster members) similar to those of the Corona Borealis supercluster 36]. These simulations show, however, that the WHIM lying in the inter-cluster regions within the supercluster produces a thermal SZE much smaller than the value observed by MITO/VSA.

\section{Cosmological Relevance}

The redshift-independent nature of the SZE allow to use this effect as a powerful cosmological probe [2, 3, [7, 12, 34, 50, by using both the redshift evolution of cluster abundance and direct probes of cosmological parameters. The SZE has a wide range of cosmological applications that we briefly review.

\subsection{Cosmological SCEnarios}

Galaxy cluster surveys are powerful tools for studying the nature of DE. In principle, the equation of state $(p=w \rho)$ parameter $w$ of the DE and its time evolution can be extracted from large solid angle, high yield surveys that can deliver tens of thousands of clusters [50]. This is possible with SZE cluster surveys like the ACT, SPT and PLANCK surveys (see Fig. 8).

The sensitivity of the cluster redshift distribution to the parameter $w$ for a large SZE cluster survey have
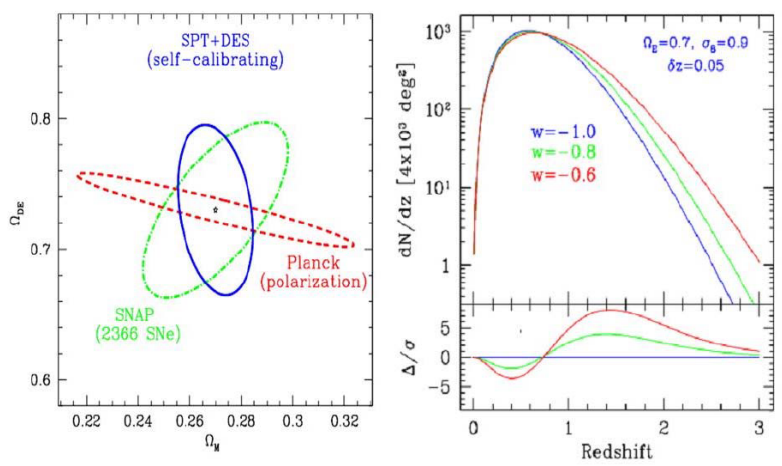

FigURE 8. Left. Forecasts for the geometry constraints from the SPT+DES galaxy cluster survey, the SNAP SNe Ia mission, and Planck. Right. The expected redshift distribution (top) and quantified differences (bottom) for models where only the DE equation of state parameter $w$ is varied. These models are normalized to produce the same local abundance of galaxy clusters. Figures from 50].

been widely discussed (see, e.g., [50]). The sensitivity to the cosmological volume dominates at intermediate redshifts $z \sim 0.5$, and the sensitivity to the growth rate dominates at $z>1$ where it is possible to disentangle more easily among various forms of the DE equation of state.

There are several requirements, which must be in place to achieve precise cosmological constraints from a cluster survey: i) a firm theoretical understanding of the formation and evolution of massive DM halos, ii) a clean and well-understood selection of large numbers ( $\gtrsim 10^{4}$ ) of massive DM halos (i.e. galaxy clusters) over a wide redshift range, iii) a cluster survey observable $Y$ that correlates with the cluster halo mass $M$ (i.e., an $M-Y$ relation), and iv) redshift estimates for each cluster.

Cluster cosmology is pursued by using a wide range of cluster observables, including the X-ray emission from the ICM, the SZE, the optical light and number of cluster galaxies, and the weak lensing shear signature of clusters. Generally speaking, cluster selection is easier, and the $M-Y$ relations are tightest in the $\mathrm{X}$-ray; there is however a strong theoretical prejudice (supported by the results of hydrodynamical simulations) that the situation will be even better at $\mathrm{mm}$ wavelengths (i.e. using SZE observations) [50].

The SZE can check for potential traces of cluster evolution better than other cosmological probes [1, 33, in particular for galaxy clusters in the medium mass regime.

In addition, new techniques are being introduced that make improved use of other less well studied cosmological tools, like, e.g., the combination of SZE and weak lensing properties of clusters. As an example, the combined SPT+DES cluster survey should provide a strong handle on the nature of the DE [50]: in this case, cluster finding and masses should arise primarily from the SZE data, and the DES optical data will 
provide photometric redshifts.

We further emphasize here that cluster surveys can be used to constrain far more general models than the constant $w$ DE models, like the time variation of the DE equation of state parameter [76, 77] or modified gravity scenarios [74].

The possibility to probe modified Gravity scenarios derives from the fact that the high-mass tail of the cluster mass function is enhanced w.r.t. the standard DE-CDM model and this yields both an increase in the number of high- $M$ cluster and modification to their cosmological evolution with redshift. Two methods have been proposed to use SZE observations to set constraints on these models: i) studies of the redshift evolution of high- $M$ clusters and of their $y$ $M$ relation [24, ii) studies of the SZE angular power spectrum using small scale CMB observation data [74. New results and tighter cosmological contraints are expected from Planck, SPT and ACT cluster surveys.

\subsection{DARK MATTER SEARCH}

Dark Matter (DM) annihilation in the halo of galaxies and galaxy clusters have relevant astrophysical implications (see e.g. 22 for a review).

Neutralinos $(\chi)$ which annihilate inside a DM halo produce quarks, leptons, vector bosons and Higgs bosons, depending on their mass and physical composition. Electrons are then produced from the decay of the final heavy fermions and bosons providing a continuous spectrum of secondaries (apart from the monochromatic electrons, with energy about $M_{\chi}$, coming from the direct channel $\chi \chi \rightarrow \mathrm{e}^{ \pm}$). The different composition of the $\chi \chi$ annihilation final state will in general affect the form of the final secondary electron spectrum [13, 17, 18.

The SZE induced by secondary electrons produced in $\mathrm{DM}(\chi)$ annihilation $\left(\mathrm{SZE}_{\mathrm{DM}}\right)$ is an unavoidable consequence of the presence and of the nature of DM in large-scale structures and its spectral shape depends on the neutralino mass and composition [15].

The presence of a substantial $\mathrm{SZE}_{\mathrm{DM}}$ is likely to dominate the overall SZ signal of a galaxy cluster at frequencies $x \gtrsim 3.8 \div 4.5$ providing a negative total SZE that is at variance w.r.t. the positive or null thermal SZE from the cluster ICM and allows to set contraints in the $\langle\sigma V\rangle-M_{\chi}$ exclusion plot for DM models [15. It is, however, necessary to stress that in such frequency range there are other possible contributions to the SZE, like the kSZE and the possible non-thermal SZE (see Fig. 1) which could provide additional biases. Nonetheless, the spectral shape of the $\mathrm{SZE}_{\mathrm{DM}}$ is quite different from that of the kSZE and of the thermal SZE and this allows to disentangle it from the overall SZE signal. An appropriate multifrequency analysis of the overall SZE based on observations performed on a wide spectral range (from the radio to the sub-mm region) is required to separate the various SZE contributions and to provide an estimate of the DM induced SZE [15]. The SZE $\mathrm{DM}_{\mathrm{DM}}$ signal also does not strongly depend on the assumed DM density profile at intermediate angular distances from the cluster center and on the DM clumpiness since $y_{\mathrm{DM}}$ is the integral of the total $P_{\mathrm{DM}}$ along the line of sight (see Eq. 2). The $\mathrm{SZE}_{\mathrm{DM}}$ is one of the cleanest probes for DM indirect search since it only depends on the DM particle nature and on the CMB photon background.

An $\mathrm{SZE}_{\mathrm{DM}}$ is expected in every DM-dominated cosmic structure from the most massive ones (galaxy clusters) to the smallest bound DM halos, thus producing negative temperature decrements (at $\nu \lesssim 400 \mathrm{GHz}$ ) in all DM halos which do not host thermal or relativistic plasma (e.g. dwarf spheroidal galaxies and/or satellites and sub-clumps of normal galaxies) and an integrated Comptonization of $\mathrm{CMB}$ radiation plus additional anisotropies in the microwave and $\mathrm{mm}$ cosmic backgrounds. This effect represents the minimum guaranteed ICS emission (from radio to sub-mm frequencies, and also at high- $E$ up to $\gamma$-rays) of DM halos in a universe dominated by $\chi$ DM.

The multi- $\nu$ nature of the DM induced emission [17, 18, 21, and the analysis of the $\mathrm{SZE}_{\mathrm{DM}}$, and the relative temperature anisotropy spectrum, provide crucial complementary probes for the presence and for the nature of DM.

\subsection{The MAGNETIZED UNIVERSE}

After recombination, primordial magnetic fields generate additional density fluctuations that enhance the number of formed galaxy clusters so that their counts and $z$-distribution can be used to set constraints on the structure of the primordial $B$-field. The results of Chandra X-ray cluster survey exclude the existence of primordial $B$ fields with amplitude larger than $\sim 1 \mathrm{nG}$ [72]. Future SZE cluster surveys have an enhanced sensitivity to constrain primordial magnetic fields because of the $z$-independence of the SZE and of the smaller biases in the cluster mass reconstruction. The Planck and SPT cluster survey have the potential to constrain a primordial $B$ fields with sub nG amplitude [72] (see Fig. 9).

\subsubsection{Cosmological Velocity Field}

The kSZE is a powerful probe to the $3 \mathrm{D}$ velocity distribution of large-scale structures and of the missing baryons distribution in the universe. However, the $\mathrm{kSZE}$ signal is overwhelmed by various contaminations and the cosmological application is hampered by loss of redshift information due to the projection effect.

A kSZE tomography method is, nonetheless, possible to alleviate these problems, with the aid of galaxy spectroscopic redshift surveys. Specifically, it has been proposed to estimate the large scale peculiar velocity through the $3 \mathrm{D}$ galaxy distribution, weigh it by the 3D galaxy density and adopt the product projected along the line of sight with a proper weighting as an estimator of the true kSZE temperature fluctuation 69]. 

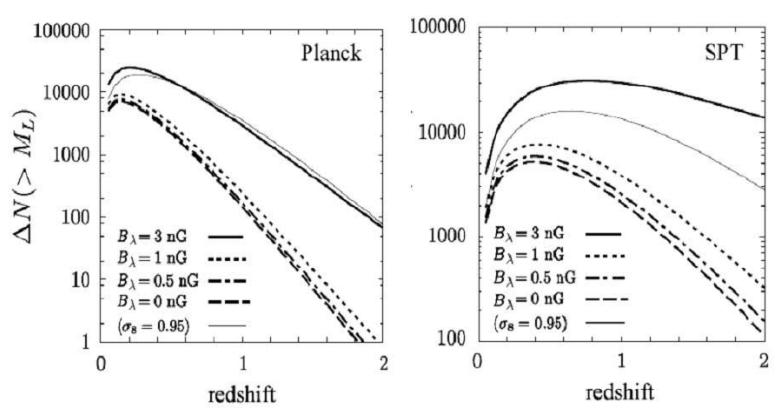

FigURE 9. The SZE cluster number counts expected for Planck and for SPT. In both panels, the solid, the dashed, the dashed-dotted and the dotted lines represent the number counts for the case of $B=3 \mathrm{nG}$, $B=1 \mathrm{nG}, B=0.5 \mathrm{nG}$ and $B=0 \mathrm{nG}$, respectively. The number counts for the $B=0 \mathrm{nG}$ and $\sigma_{8}=0.95$ (corresponding to $\sigma_{8}$ for $B=3 \mathrm{nG}$ ) is also shown (thin solid line) for comparison. Figure from [72].

First evidence for a non-zero mean pairwise cluster momentum due to the kSZE signal was obtained by ACT [38, but it can also be interpreted as a measure of baryons on cluster length scales.

\subsection{Other Cosmological applications}

Detailed models of structure formation processes and reionization history of the universe (including a consistent description of galaxies, quasars and matter density fluctuations) allow to compute the secondary CMB anisotropies generated by the kSZE [75]. The contribution due to patchy reionization seems however to be negligible except at large angular scales $(\theta \gtrsim 3.5$ arcmin $)$ and small wavenumbers $\left(\ell<10^{3}\right)$. Over this range, which corresponds to scales larger than the typical size of HII regions, the signal actually comes from the cross-correlation of ionized bubbles, induced by the correlations of the rare radiation sources. On smaller scales, the inter-galactic medium (IGM) contribution is governed by the fluctuations of the matter density field itself. However, over this range the secondary anisotropies should be dominated by the contribution from galactic halos, which are characterized by smaller scales than the IGM (and larger densities). This leads to a cutoff of the CMB anisotropy power-spectrum at a large wavenumber $\ell \sim 10^{6}$, while at low wavenumbers $\ell<10^{3}$ a white noise power-spectrum is recovered.

Thus, observations of these secondary CMB anisotropies should probe the correlation properties of the underlying matter density field, through the correlations of the HII regions and the small-scale density fluctuations.

The (thermal and kinematic) SZE and its polarization are important tools to test the homogeneity of our universe through probes of the Copernican Principle $(\mathrm{CP})$ 46. In fact, large variations of the thermal SZE induced by CMB photons that have temperature significantly different from the blackbody temperature we observe directly, and that arrive at galaxy cluster from points inside our light cone, could indicate a violation of the $\mathrm{CP}$ and homogeneity. Analogously, large variations of the $\mathrm{CMB}$ dipole measured by the cluster kSZE could also indicate a violation of the $\mathrm{CP}$ and homogeneity. The SZE polarization contains more refined information on the CMB temperature and thus it can also provide a powerful probe of $\mathrm{CP}$ and homogeneity. Thus, observations of large SZE and of its polarization w.r.t. to the expectations of the SZE produced from a pure blackbody CMB spectrum might provide indications of a non-FLRW universe.

Finally, the evolution of the CMB temperature can be probed out to substantial redshifts by combining SZE observations of galaxy clusters [45] (setting constraints at low-moderate $z$ ) with SZE observations of RG lobes (able to set constraints even at high $z$ ) 20 . These data will provide further constraints on the energy release mechanisms possibly occurring at early cosmic ages.

\section{Future DiRECTIONS}

The impact of SZE observation for astrophysical and cosmological application is steadily increasing since the advent of dedicated experiments and large scale surveys in the microwave and $\mathrm{mm}$ frequency range. The quality and the spatial resolution of SZE images is reaching the level of X-ray images and the spectral coverage in systematically extending in the $\mathrm{mm}$ region. There is a strong theoretical motivation (supported by analytical calculations, hydro-dynamical simulations and some preliminary data) that the situation will be even better extending the SZE study at sub-mm wavelengths, where highest spatial resolutions can be achieved with a wide spectral coverage able to decipher the physical details of the electron distribution in the atmosphere of cosmic structures.

New paths of theoretical investigations are underway and concern both the detailed study of ICS mechanism in cosmic atmospheres and the impact of the various plasma structure and fields in cosmic structures. The possibility to perform precise measurements of the various SZE signals and to extract the relevant astrophysical information depends crucially on the capability to have spatially-resolved spectral observations of SZE sources over a wide $\nu$ band, from radio to sub-mm. In particular, the important condition for such study is to have a wide-band continuum spectroscopy and especially a good spectral coverage and sensitivity in the high-frequency band, where most of the astrophysical effects reveals more clearly. Spatially resolved spectroscopic and polarimetric observations of the SZE in the frequency range from $\sim 100 \mathrm{GHz}$ to $\sim 1 \mathrm{THz}$ are the key to improve our understanding of the structure of cosmic atmospheres through analysis of the intensity and polarized SZE signal, and will allow to use this technique to probe the structure of the universe. 


\section{ACKNOWLEDGEMENTS}

S.C. acknowledges useful and enriching discussions with P. De Bernardis, V. Dogiel, R. Maartens, P. Marchegiani, S. Masi and D. Prokhorov. S.C. acknowledges support by the South African Research Chairs Initiative of the Department of Science and Technology and National Research Foundation and by the Square Kilometre Array (SKA).

\section{REFERENCES}

[1] Ade, P. et al. 2011, arXiv:1101.2024

[2] Aghanim, N. et al., 1997, A\&A, 325, 9

[3] Barbosa, D. et al., 1996, A\&A, 314, 14

[4] Battistelli, E. et al. 2006, ApJ, 645, 826

[5] Baumann, D. \& Cooray, A. 2003, New Astron.Rev. 47, 839

[6] Benson, B.A. et al. 2004, ApJ, 617, 829

[7] Birkinshaw, M., 1999, Physics Reports, 310, 97

[8] Birzan, L. et al. 2004, ApJ, 607, 800

[9] Blundell, K.M., et al. 2006, ApJ, 644, L13

[10] Bonometto, S. et al. 1970, A\&A, 7, 292

[11] Challinor, A. \& Lasenby, A. 1998, ApJ, 499, 1

[12] Colafrancesco, S. et al. 1997, ApJ, 479, 1

[13] Colafrancesco, S. \& Mele, B. 2001, ApJ, 562, 24

[14] Colafrancesco, S., Marchegiani, P. \& Palladino, E. 2003, A\&A, 397, 27

[15] Colafrancesco, S. 2004, A\&A, 422, L23

[16] Colafrancesco, S. 2005, A\&A, 435, L9

[17] Colafrancesco, S., Profumo, S. \& Ullio, P.2006, A\&A, 455,21

[18] Colafrancesco, S., Profumo, S. \& Ullio, P. 2007, PhRvD, 75, 3513

[19] Colafrancesco, S., 2007, New Astronomy Reviews, 51, 394

[20] Colafrancesco, S. 2008, MNRAS, 385, 2041

[21] Colafrancesco, S. 2010, in "Multi-frequency behaviour of high-energy cosmic structures", MmSAIt, 81, 104

[22] Colafrancesco, S. 2010, in "Astrophysics and cosmology after Gamow", 2010, AIPC, 1206, 5

[23] Colafrancesco, S. \& Marchegiani, P., 2010, A\&A, 520, 31

[24] Colafrancesco, S., Marchegiani, P. \& Buonanno, R. 2011, A\&A, 527, L1

[25] Colafrancesco, S. \& Marchegiani, P., 2011, A\&A, 535, 108

[26] Colafrancesco, S., Marchegiani, P., De Bernardis, P. \& Masi, S. 2012, A\&A in press

[27] Colafrancesco, S. \& Tullio, M. 2012, preprint

[28] Colafrancesco, S., et al. 2011, in preparation

[29] The COrE collaboration 2011, A White Paper, arXiv:1102.2181

[30] Croston, J.H. et al. 2005, ApJ, 626, 733

[31] De Bernardis, P., Colafrancesco, S. et al. 2012, A\&A, 538, 86
[32] De Petris, M. et al. 2002, ApJ, 574, L119

[33] Delsart, P., Barbosa, D. \& Blanchard, A. 2010, A\&A, 524,81

[34] Diego, J.M, Martinez, E., Sanz, J.L., Benitez, N. \& Silk, J. 2002, MNRAS, 331, 556

[35] Dogiel, V., Colafrancesco, S., Ko, C.M. et al. 2007, A\&A, 461, 433

[36] Flores-Cacho, I. et al. 2009, MNRAS, 400, 1868

[37] Genova-Santos, R. et al. 2008, MNRAS, 391, 1127

[38] Hand, N. et al. 2012, arXiv:1203.4219

[39] Harris, D.E. \& Krawczynski, H. 2002, ApJ, 565, 244

[40] Itoh, N., Kohyama, Y. \& Nozawa, S. 1998, ApJ, 502, 7

[41] Kataoka, J. et al. 2003, A\&A, 410, 833

[42] Kompaneets, A.S. 1957, Soviet Phys. JETP, 4, 730

[43] Lamarre, F. et al. 1998, ApJ, 507, L5

[44] Lavaux, G. et al. 2004, MNRAS, 347, 729

[45] Luzzi, G. et al. 2009, ApJ, 705, 1122

[46] Maartes, R. 2011, arXiv:1104.1300

[47] McNamara, B. R. et al. 2000, AAS, 32.13211

[48] McNamara, B.R. \& Nulsen, P.E.J. 2007, arXiv:0709.2152

[49] Million, E. T., \& Allen, S. 2009, MNRAS, 399, 1307

[50] Mohr, J. 2004, in "Observing Dark Energy" arXiv:astro-ph/0408484v1

[51] Nozawa, S., Kohyama, Y. \& Itoh, N. 2010a, arXiv:1009.2311

[52] Nozawa, S., Kohyama, Y. \& Itoh, N. 2010b, PhRvD, 82,3009

[53] Pfrommer, C., Ensslin, T., \& Sarazin, C. L. 2005, A\&A, 430, 799

[54] Phillips, P.R. 1995, ApJ, 455, 419

[55] Planck Collaboration, 2011, A\&A 536, A8

[56] Planck Collaboration 2011, A\&A 536, A9

[57] Planck Collaboration 2011, A\&A 536, A10

[58] Planck Collaboration 2011, A\&A 536, A911

[59] Planck Collaboration 2011, A\&A 536, A12

[60] Planck Collaboration 2011, arXiv:1112.5595P

[61] Pointecouteau, E. 2012, in Proceeding of the Moriond Meeting 'Cosmology'

[62] Prokhorov, D., Antonuccio-Delogu, V. \& Silk, J. 2010, arXiv:1006.2564

[63] Prokhorov, D., Colafrancesco, S. et al. 2011a, A\&A, 529, 39

[64] Prokhorov, D., Colafrancesco, S. et al. 2011b, MNRAS, 416, 302

[65] Prokhorov, D. and Colafrancesco, S., 2012, MNRAS, 424, L49

[66] Rephaeli, Y. \& Lahav, O. 1991, ApJ, 372, 21

[67] Rowe, B. \& Silk, J. 2010, MNRAS in press, arXiv:1005.4234v2

[68] Sazonov, S. \& Sunyaev, R. 1999, MNRAS, 310, 765 
[69] Shao, J. et al. 2010, arXiv:1004.1301

[70] Sunyaev, R.A. \& Zel'dovich, Y.B.: Comm. Astrophys. Space Phys. 1972, 4, 173

[71] Sunyaev, R. A. \& Zeldovich, Ia. B. 1980, ARA\&A, 18,537

[72] Tashiro, H. et al. 2010, arXiv:1010.4407v1

[73] Taylor, J.E. et al. 2003, MNRAS, 345, 1127

[74] Tsutomu, K. \& Hiroyuki, T. 2009, MNRAS, 398, 477

[75] Valageas, P., Balbi. A. \& Silk, J. 2001, A\&A, 367, 1
[76] Wang, S. et al. 2004, Ph.Rev.D., 70, 123008

[77] Weller, J., Battye, R. A., \& Kneissl, R. 2002, Phys. Rev. Lett., 88, 231301

[78] Wolfe, B. \& Melia, F. 2006, ApJ, 638, 125

[79] Wolfe, B. \& Melia, F. 2008, ApJ, 687, 193

[80] Yamada, M. et al. 2010, AJ, 139, 2494

[81] Zemcov, M. et al. 2010, A\&A, 518, L16 